\title{
Breite Datenbasis für die IL-17A-Inhibition bei ankylosierender Spondylitis
}

Dass der Interleukin (IL)-17-Signalweg bei axialen Spondylarthritiden eine wichtige Rolle spielt, ist für Secukinumab bereits gut belegt. Beim ACR wurden nun überzeugende Daten auch für Ixekizumab vorgestellt. Der Dritte im Bunde - bisher nur mit Daten zur PsA - könnte der IL-17A+F-Inhibitor Bimekizumab werden.

Für den ersten in der Indikation ankylosierende Spondylitis (AS) zugelassenen Interleukin(IL)-17A-Inhibitor Secukinumab wurden in Chicago die abschließenden Daten der MEASURE-1- und 2-Studien vorgestellt. PD Dr. Xenophon Baraliakos, Rheumazentrum Herne, berichtete die 5-Jahres-Daten zur Effektivität und Sicherheit aus der Extensionsphase von MEASURE 1 (Abstract L13).

\section{Daten zu Secukinumab}

Sie belegen eine stabil über fünf Jahre hinweg anhaltende Wirksamkeit auf multiple Endpunkte, darunter klinische Zeichen und Symptome, körperliche Funktion und objektive Marker der Entzündung. Die Wirksamkeit konnte durch eine Dosiseskalation von $75 \mathrm{mg}$ auf $150 \mathrm{mg}$ Secukinumab gesteigert werden. Die langjährige Therapie mit dem IL-17A-Inhibitor wurde gut vertragen; neue Sicherheitssignale traten nicht auf.

\) Die Therapie mit Secukinumab wirkte sich positiv auf die Knochendichte aus

Auch auf die radiologische Progression wirkte sich Secukinumab positiv aus. Wie unter anderem der Vergleich der 2-Jahres-Daten aus der MEASURE 1-Studie mit denen der historischen Kohorte ENRADAS ergab, kam es unter dem Biologikum zu einer numerisch geringeren Veränderung des mSASSS (modified Stoke Ankylosing Spondylitis Spinal Score) gegenüber dem Ausgangswert; sie betrug 0,55 versus 0,89 $(p=0,1852)$ (Abstract 670).
Der Anteil der Patienten ohne radiologische Progression (mSASSS-Veränderung nach 2 Jahren $\leq 0, \leq 0,5, \leq 1$ und $\leq 2$ ) war in der MEASURE 1-Kohorte zu allen Messpunkten numerisch höher als in der Biologika-naiven Kohorte. Positiv wirkte sich die Therapie mit Secukinumab auch auf die Knochendichte, insbesondere der Lendenwirbelsäule aus (Abstract 1597).

》) Der IL-17A+F-Inhibitor Bemikizumab konnte seine Wirksamkeit in der BE ACTIVE Studie beweisen

In der Abschlussuntersuchung der MEASURE 2-Studie nach vier Jahren fand sich für die $150 \mathrm{mg}$-Dosis, unabhängig von einer Vorbehandlung mit Tumor Nekrose Faktor Inhibitoren (TNFI), nicht nur eine weiterhin gute Wirksamkeit für alle Endpunkte, sondern auch ein unverändert günstiges Sicherheitsprofil und eine hohe Therapietreue der Patienten (Abstract 2556).

\section{Auch Ixekizumab bei AS wirksam}

Wie Prof. Dr. Desirée van der Heijde, Universität Leiden, in Chicago berichtete, kann Ixekizumab (IXE) eine aktive radiologische axiale Spondyloarthritis $(r-a x-S p A)$ von Biologika-naiven Patienten effektiv lindern (Abstract 1864). In der Studie COAST V wurde die Effektivität des IL-17A-Inhibitors in einer zwei- und einer vierwöchentlichen Gabe von 80 mg gegen Adalimumab und Placebo geprüft. Den primären Endpunkt eines ASAS40Ansprechen nach 16 Wochen erzielten unter beiden IXE-Dosierungen signifi- kant mehr Patienten als unter Placebo. Die ASAS40-Ansprechraten betrugen $48 \%$ beim längeren Dosisintervall und $52 \%$ beim kürzeren versus $18 \%$ unter Placebo und $36 \%$ unter Adalimumab.

Aufgrund des schnellen Wirkeintritts fand sich beim ASAS20-Ansprechen bereits nach einer Woche ein signifikanter Unterschied gegenüber Placebo, für das ASAS40-Ansprechen nach zwei Wochen. Ähnlich überzeugend waren die Ergebnisse für sekundäre Endpunkte, wie gesundheitsbezogene Lebensqualität, Entzündungsmarker und Bildgebung, sowie für die globale Funktion (Abstract 1865). Es traten keine unerwarteten Sicherheitssignale auf.

Bei Patienten mit Psoriasis-Arthritis konnte der IL-17A+F-Inhibitor Bemikizumab, der vielleicht bald Dritte im Bunde der IL-17-Inhibitoren, seine Wirksamkeit in der BE ACTIVE Studie unter Beweis stellen.

rheuma plus $2019 \cdot 18: 88$ https://doi.org/10.1007/s12688019-0248-9

(c) Springer-Verlag GmbH Austria, ein Teil von Springer Nature 2019 